\title{
Window length selection of singular spectrum analysis and application to precipitation time series
}

\author{
Mingdong Sun* and Xuyong Li \\ State Key Laboratory of Urban and Regional Ecology, \\ Research Center for Eco-Environmental Sciences, \\ Chinese Academy of Sciences, Beijing, 100085 \\ Received: 28/08/2016, Accepted: 30/11/2016, Available online: 05/10/2017 \\ *to whom all correspondence should be addressed: mingdongsun@hotmail.com
}

\section{Abstract}

Window length is a very critical tuning parameter in Singular Spectrum Analysis (SSA) technique. For finding the optimal value of window length in SSA application, Periodogram analysis method with SSA for referencing on the selection of window length and confirm that the periodogram analysis can provide a good option for window length selection in the application of SSA. Several potential periods of Florida precipitation data are firstly obtained using periodogram analysis method. The SSA technique is applied to precipitation data with different window length as the period and experiential recommendation to extract the precipitation time series, which determines the leading components for reconstructing the precipitation and forecast respectively. A regressive model linear recurrent formula (LRF) model is used to discover physically evolution with the SSA modes of precipitation variability. Precipitation forecasts are deduced from SSA patterns and compared with observed precipitation. Comparison of forecasting results with observed precipitation indicates that the forecasts with window length of $L=60$ have the better performance among all. Our findings successfully confirm that the periodogram analysis can provide a good option for window length selection in the application of SSA and presents a detailed physical explanation on the varying conditions of precipitation variables.
Keywords: Singular spectrum analysis (SSA), Window Length, Periodogram Analysis, Linear Recurrent Formula (LRF).

\section{Introduction}

Singular Spectrum Analysis (SSA) is an innovative and reliable technique for time series analysis in many scientific research fields. Since firstly proposed by Broomhead and King (1986a); Broomhead and King (1986b) and Broomhead et al. (1987) in the publication, this method has attracted extensive attention in different areas, such as climatology, meteorology and geophysical analysis (Ghil and Vautard, 1991; Vautard and Ghil, 1989; Yiou et al., 1996).

There have been many research studies on forecasting and simulation in individual interested fields with statistical methods and hydrological model(Chen et al., 2015; Gholami et al., 2015; Nourani et al., 2008; Sun and Kim, 2016; Taormina and Chau, 2015; Wang et al., 2015; Wu et al., 2009). Much research has been devoted to the methodological aspects and application of the SSA technique, which prove that SSA is a useful tool in various applied areas with analysis of diverse unitary and multivariate time series. Vautard et al., (1992) showed that SSA provided an unrefined but powerful approximation which worked well for short and noisy time series in applications to geophysical data. Allen and Smith (1996) illustrated the basic format of SSA with an investigation on regulation oscillations through exploring 
some variations on the Monte Carlo SSA algorithm, and considered that SSA was suitable for multivariate series. Using multi-scale concept from wavelet analysis, Yiou et al. (2000) extended the SSA method to non-stationary time series including intermittent variance divergence. In astrophysical applications, Varadi et al. (1999) explored a method with SSA for detecting the low-amplitude solar oscillations with long and noisy time series. Hassani (2007) compared the performance of the SSA technique with those of Seasonal Autoregressive Integrated Moving Average (SARIMA) model, plural Autoregression (ARAR) algorithm and Holt-Winter algorithm on the accidental deaths time series data in the USA, and confirmed more accurate forecasting of the SSA technique. Marques et al. (2006) applied the SSA method to some unitary hydrological time series, and demonstrated its ability of decomposing primary information and the related forecasting advantages. Baratta et al. (2003) proposed a new development about the application of SSA, and forecasted the separate rainfall intensity time series in the Tiber basin. Hassani and Zhigljavsky (2009) described the methodology of SSA and exhibited that SSA was also a very useful method for the analysis and forecasting of economic time series. Alonso et al. (2005) showed that SSA had some unique advantages in biomechanical analysis, as a digital filtering method to remove the noise.

Based on the general structure of the algorithm underlying SSA, there are two basic and important parameters, i.e., the window length $L$ and the number of eigentriples $r$ in the whole procedure of the SSA technique. Appropriate choice of $L$ and $r$ can result in an effective decomposition of time series. Obviously, the selection of parameters $L$ and $r$ mainly depends on the data to be analyzed. Besides, some worthy work and several techniques can be used to select appropriate values of parameters. Elsner and Tsonis (1996) gave some discussion and remark about choosing parameters of SSA with common practice. Hassani et al. (2011) analyzed the theory of separability between the modulated signal and the noise component, and determined the optimal value of window length in SSA. Golyandina (2010) compared the related and particular characteristics of SSA with subspace-based methods and gave some recommendations on the selection of parameters.
Considering theoretical extrapolation, window length $L$ should be large enough but less than half of time series length (Golyandina et al., (2001). Larger value of $L$ makes longer period oscillations to be solved, but too large value of $L$ may involve a large number of eigentriples and miss some important principal components with high contributions. It was noticed that the variation of $L$ could influence the separability feature of reconstructed components in the SSA technique. In some recent studies, window length $L$ was chosen from experience (Marques et al., 2006), or repeatedly tried with varying window length (Chau and $\mathrm{Wu}, 2010$ ), or taken as proportional of data length, like N/3, N/4 (Hassani and Zhigljavsky, 2009). Although lots of trial applications and various methods have been discussed for the selection of optimal value of $L$, there is still a lack of theoretical regulation for window length choosing. This study mainly focuses on the analysis of the most dominant component of the seasonal cycle and evolution of the precipitation variable. Periodogram analysis method with SSA for referencing on the selection of window length and confirm that the periodogram analysis can provide a good option for window length selection in the application of SSA. A regressive model linear recurrent formula (LRF) model is used to discover physically evolution with the SSA modes of precipitation variability. Precipitation forecasts are deduced from SSA patterns. This study presents a detailed physical explanation on the varying conditions of precipitation variables and explores a good and useful statistical method for singular precipitation time series forecasting.

\section{Methodology and Data}

The SSA method is particularly significant to extracting the essential characteristics of time series. Based on the component time series and considering the effect of SSA, Linear Recurrent Formula (LRF) is applied to forecast the component time series.

\subsection{Singular Spectrum Analysis}

The primary purpose of SSA is to decompose the original series into a few component series group where each component can be distinguished as a tendency component, periodic or quasi-periodic component and noise. The main descriptions of the SSA algorithm follow the methodology in Golyandina et al. (2001). 
The SSA technique contains four continuous steps which are related to two stages: embedding and singular value decomposition related to the decomposition stage including singular value decomposition (SVD), and grouping and diagonal averaging related to the reconstruction stage.

\subsubsection{Decomposition}

Step 1: Embedding

The embedding step projects the original time series to a sequence of multidimensional lagged vectors.

Assume there is a nonzero time series $X=\left(x_{1}, x_{2}, \ldots, x_{N}\right)$ with a length of $N$. Let window length $L$ be an integer $(1<L<N)$, and then the embedding procedure forms $K=N-L+1$ lagged vectors, $Y_{i}=\left(x_{i}, \ldots, x_{i+L-1}\right)^{T}, 1 \leq i \leq K . Y_{i}$ is an $L$-lagged vector with dimension $L$, and thus the $L$-trajectory matrix of the series $X$ is $Y=\left[Y_{1}, Y_{2}, \ldots, Y_{K}\right]$, which has lagged vectors as its columns. That is to say, the trajectory matrix $(L \times K)$ is

$$
\mathrm{Y}=\left[\mathrm{Y}_{1}, \mathrm{Y}_{2}, \ldots, \mathrm{Y}_{\mathrm{K}}\right]=\left[\begin{array}{cccc}
\mathrm{x}_{1} & \mathrm{x}_{2} & \cdots & \mathrm{x}_{\mathrm{K}} \\
\mathrm{x}_{2} & \mathrm{x}_{3} & \cdots & \mathrm{x}_{\mathrm{K}+1} \\
\mathrm{x}_{3} & \mathrm{x}_{4} & \cdots & \mathrm{x}_{\mathrm{K}+2} \\
\vdots & \vdots & \ddots & \vdots \\
\mathrm{x}_{\mathrm{L}} & \mathrm{x}_{\mathrm{L}+1} & \cdots & \mathrm{x}_{\mathrm{N}}
\end{array}\right]
$$

The trajectory matrix $Y$ has equal elements on the diagonals $(i+j=$ constant). Thus, the trajectory matrix is corresponding to the time series when $N$ and $L$ are fixed.

Step 2: Singular value decomposition

This step refers to singular value decomposition (SVD) of the trajectory matrix.

Let $S=Y Y^{T}$. Denote the eigenvalues of matrix $S$ by $\lambda_{1}, \ldots, \lambda_{L}$ in the decreasing order of magnitude (i.e., $\lambda_{1} \geq \ldots \geq \lambda_{L} \geq 0$ ), and the orthonormal system of the eigenvectors corresponding to these eigenvalues by $U_{1}, \ldots, U_{L}$. Let $d$ be the number of nonzero eigenvalues, $0<d \leq L$. Denote that $\mathrm{V}_{\mathrm{i}}=\mathrm{Y}^{\top} \mathrm{U}_{\mathrm{i}} / \sqrt{\lambda_{\mathrm{i}}}(i=1, \ldots, d)$, and the elementary matrices can be given by $X_{i}=\sqrt{\lambda_{\mathrm{i}}} \mathrm{U}_{\mathrm{i}} \mathrm{V}_{\mathrm{i}}^{\top}$ Thus, SVD of the trajectory matrix $Y$ can be written as: $Y=X_{1}+X_{2}+\ldots+X_{d}$. Obviously, the contribution of the first matrices to the norm of $Y$ is much higher than that of the last matrices.

\section{Step 3: Grouping}

The grouping step corresponds to splitting the elementary matrices $X_{i}$ into several groups and summing the matrices within each group.

Separate the set of indices $\{1, \ldots, d\}$ into $r$ disjoint subsets $I_{1}, \ldots, I_{r}$, and let $I=\left\{i_{1}, \ldots, i_{p}\right\}$. Then, the resultant matrix $Y_{l}$ corresponding to the group $I$ can be defined as $Y_{l}=X_{i 1}+\ldots+X_{i p}$. These matrices are computed for $I=I_{1}, \ldots, I_{r}$, and finally achieve the decomposition of $Y=X_{11}+\ldots+X_{I r}$. By the way, the procedure of choosing the sets $I_{1}, \ldots, I_{r}$ is called as eigentriple grouping.

\section{Step 4: Diagonal averaging}

In the last step, each elementary matrix of the grouped decomposition is transformed into a new principal component series with a length of $N$. Let $e_{i j}$ be any element of the elementary matrices $X_{i}$ with $L \times K$ dimension, $1 \leq i \leq L, 1 \leq j \leq K$. Set $L^{*}=\min (L, K), K^{*}=\max (L, K)$ and $N=L+K-1$. Let $e^{*}{ }_{i j}=e_{i j}$ if $L<K$, and $e^{*}{ }_{i j}=e_{j i}$ otherwise. Diagonal averaging can transfer the matrix $X_{i}$ to the series $f_{1}, \ldots, f_{N}$ by the following formula:

$$
f_{k}=\left\{\begin{array}{cc}
\frac{1}{k} \sum_{r=1}^{k} e_{r, k-r+1}^{*} & \text { for } 1 \leq k<L^{*} \\
\frac{1}{L^{*}} \sum_{r=1}^{L^{*}} e_{r, k-r+1}^{*} & \text { for } L^{*} \leq k \leq K^{*} \\
\frac{1}{N-k+1} \sum_{r=k-K^{*}+1}^{N-k+1} e_{r, k-r+1}^{*} & \text { for } K^{*}<k \leq N
\end{array}\right.
$$

The above expression corresponds to the average of matrix elements over the 'diagonals' $i+j=k+1$. Besides, it is necessary to point out that the application of the SSA algorithm needs to select the values of two parameters: the window length $L$ and the number $r$.

\subsubsection{Separability}

The main feature of SSA is that it can well separate a time series into different components. So, the original series can be decomposed successfully only if the resultant components of the series are relatively separable from each other. The separability characteristic of two reconstructed component series $F^{(1)}$ and $F^{(2)}$ can be quantitatively measured by the weighted correlation or $w$-correlation $\rho$ :

\subsubsection{Reconstruction}


$\rho_{12}^{(w)}=\frac{\left(F^{(1)}, F^{(2)}\right)_{w}}{\left\|F^{(1)}\right\|_{w}\left\|F^{(2)}\right\|_{w}}$

where ||$F^{(i)} \mid I_{w}=\sqrt{\left(F^{(i)}, F^{(i)}\right)_{w}},\left(F^{(i)}, F^{(j)}\right)_{w}=\sum_{k=1}^{N} w_{k} f_{k}^{(i)} f_{k}^{(j)},(i, j=1,2), w k=\min \{k, L, N-k\}$.

If the absolute value of the $w$-correlation in reconstructed components is small, then the two corresponding component series are considered to be separable; otherwise the components should probably be grouped together. This trend is similar to that in SSA.

\subsection{Periodogram Analysis}

Periodogram is a nonparametric estimate of the power spectral density (PSD) during a wide-sense stationary random process. The phrase of PSD was specially proposed for representation of a variable quantity which corresponded to the spectrum (Schuster, 1989).

Periodogram analysis of a series can determine what frequency is included in the series. For a sequence $\left(x_{1}, x_{2}\right.$, ..., $\left.x_{N}\right)$, the definition of periodogram can be described as follows:

$P(f)=\frac{1}{N}\left|\sum_{n=1}^{N} x(n) e^{-i 2 \pi f n}\right|^{2}$

where $P$ is the estimation of spectral density and $f$ is the embedded frequency of series.

Substituting the period $\frac{1}{T}$ for $f$, Eq. (4) can be rewritten as:

$P(T)=\frac{1}{N}\left|\sum_{n=1}^{N} x(n) e^{\frac{-i 2 \pi n}{T}}\right|^{2}$

Eq. (5) shows the relationship between estimation of spectral density and embedded period of the series. It can tell us what period should be considered and regarded as the primary period.

\subsection{Linear Recurrent Formula (LRF)}

The theory of LRF and the related characteristic polynomials are well known and widely used. The details can be found in Golyandina et al. (2001).
According to its definition, a nonzero series $X_{N}=\left(x_{1}, x_{2} \ldots\right.$, $x_{N}$ ) is governed by LRF with dimension not exceeding $d \geq 1$ if

$x_{i+d}=\sum_{k=1}^{d} a_{k} x_{i+d-k}$

where $a_{1}, \ldots$, and $a_{d}$ satisfy $a_{d} \neq 0$, and $0 \leq i \leq N-d+1$.

LRF can be used in both theoretical and empirical analysis. It can turn a series data into a broad various model depend on different lagged variables.

\subsection{Data Description}

The data in the present study are the average monthly precipitation data in Florida statewide from January 1981 to December 2014. The data are provided by National Centers for Environmental Information (NCEI) of National Oceanic and Atmospheric Administration (NOAA) which saves the world's largest climate data archive and provides climatological services and data in United States. In order to ensure stationary of precipitation time series, data are checked using Dicky Fuller test before starting the research. Fig. 1 shows the singular time series data of monthly precipitation in 34 years (1981-2015), of which records of 30 years (1981-2010) are used for analysis, and the remaining of 5 years are used to validate the forecasting. Visual analysis of Fig. 1 clearly presents a yearly harmonic component.

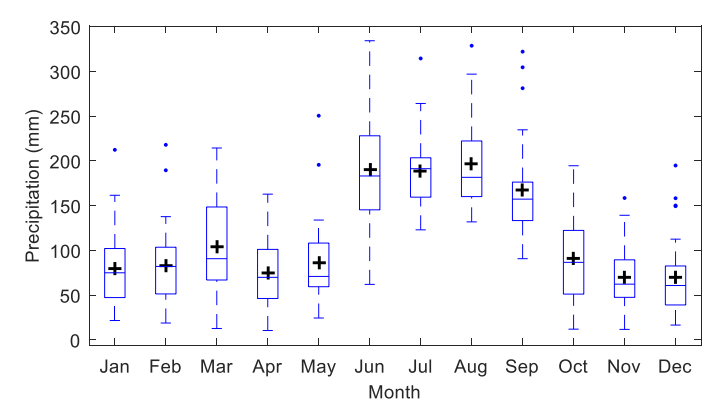

Figure 1. Boxplots of monthly precipitation data totals from 1981 to 2015 in Florida 


\section{Results and Discussion}

\subsection{Analysis}

Florida monthly precipitation data for 30 years (1981-2010) are tested in this study, and various window lengths are selected in order to describe the extensive performance of the precipitation time series and meanwhile consider the proportionality to the possible period. To find the possible embedded periods of given data, periodogram analysis is applied to the original data, and the periodogram plot is shown in Fig. 2. As can be seen, four inherent periods are prominent in the figure, corresponding approximately to the periods of $T_{1}=12$, $T_{2}=20, T_{3}=42$ and $T_{4}=126$. These inherent possible periods are considered as the options for the values of window length.

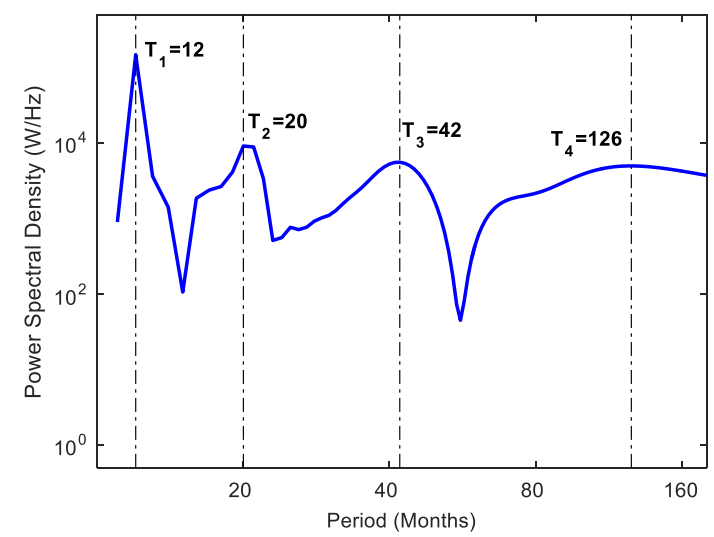

Figure 2. Periodogram plot of precipitation time series

Then, the SSA technique is applied to the tested data of the 30 years. The main motivation for employing this technique is to extract the various trends from precipitation time series and further forecast the subsequent continuous components.

The length $N$ of the tested data is 360 , and we take $N / 2=180, N / 3=120, N / 6=60$ and $N / 12=30$ as window lengths. With consideration of these selected window lengths and the SVD of the trajectory matrix, several bunch components are obtained and ordered according to their contributions to the decomposition. Fig. 3 displays the first leading principal components and the corresponding contribution percentages obtained from analysis. The variation trends change from fluctuation to smooth with increasing window length, and the corresponding contribution percentages are gradually decreased.

As mentioned above, the main function of SSA is to decompose the original series into an identified trend (periodic or quasi-periodic) components from noise components. As shown in Fig. 4 and Fig. 5, each reconstructed component is shown. According to different window lengths, the 2nd-3rd reconstructed component pairs present annual oscillation; the 4th-5th component pairs present semiannual oscillation; the 6th-7th component pairs present quarterly oscillation; and so on. All of these reconstructed components will be used to assess its forecasts by the LRF.

Fig. 6 shows the absolute values of $w$-correlations for reconstructed components in a grey scale corresponding to the values of 0 to 1 with different window lengths. In Fig. 6, the adjacent reconstructed components are mostly high correlations, belonging to one group and containing periodic alike oscillation with similar contribution percentages. The results confirm the grouping feature of those leading components as shown in Fig. 4 and Fig. 5. In most cases, the components with less percentage are corresponding to noise components in the series. Fig. 6 also shows the set of leading components. Base on the $w$-correlations, the leading components numbers are taken among different window length. If the correlations are high below the line of numbers, these components are well separated from a block of the remaining components; otherwise if the correlations are messy over the line, these reconstructed components are possibly considered as noise components.

Hence, these leading components can properly describe the general tendency of the series.

Table 1. Summary of reconstruction time series

\begin{tabular}{c|c|c|c}
\hline $\begin{array}{c}\text { Window } \\
\text { Length }\end{array}$ & $\begin{array}{c}\text { Leading } \\
\text { Components }\end{array}$ & $\begin{array}{c}\text { Contribution } \\
\text { Percent (\%) }\end{array}$ & RMSE \\
\hline 30 & 10 & 92.71 & 32.75 \\
60 & 15 & 92.66 & 34.23 \\
120 & 17 & 91.41 & 38.09 \\
180 & 24 & 92.41 & 36.59 \\
\hline 12 & 5 & 92.72 & 35.00 \\
20 & 7 & 92.33 & 33.79 \\
42 & 13 & 92.90 & 33.09 \\
126 & 19 & 91.73 & 37.38 \\
\hline
\end{tabular}



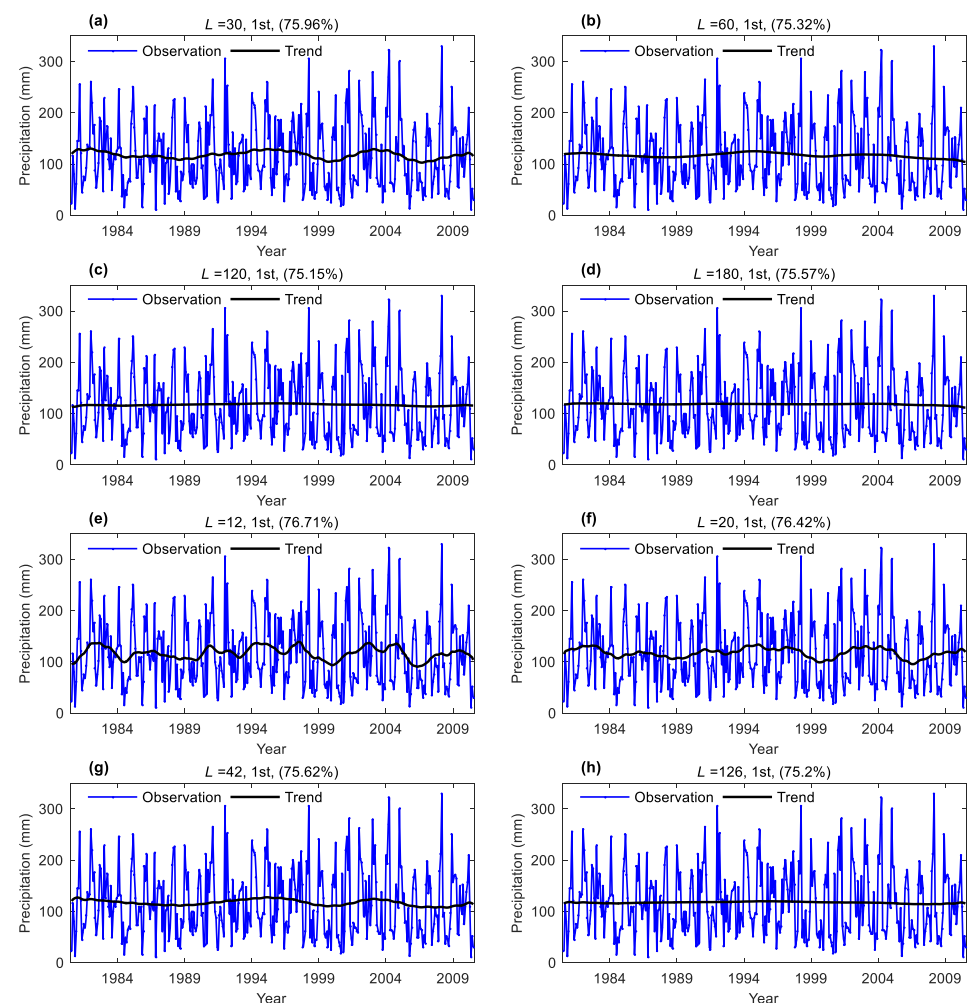

Figure 3. Observation and 1st leading components with related contributions percent
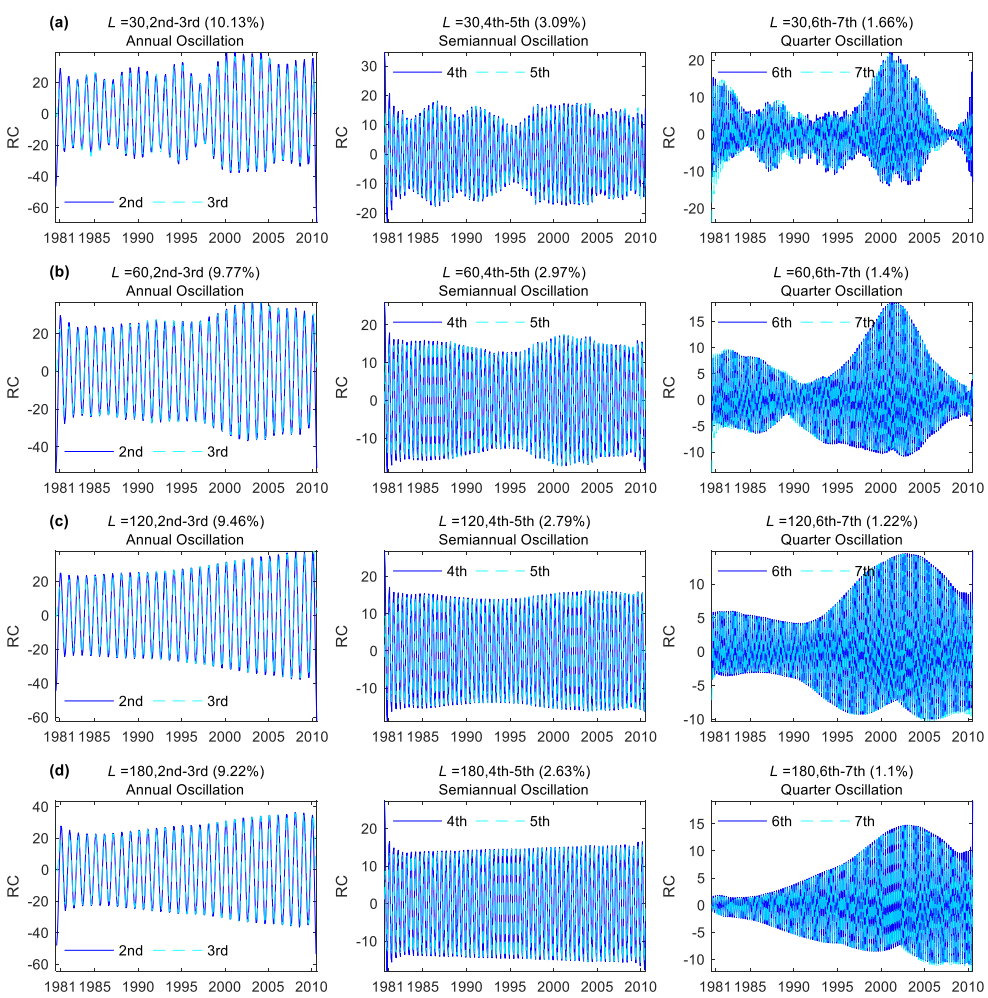

Figure 4. Annual, semiannual and quarter oscillation components are reconstructed from components pairs with $L$ value as $30,60,120$ and 180. 

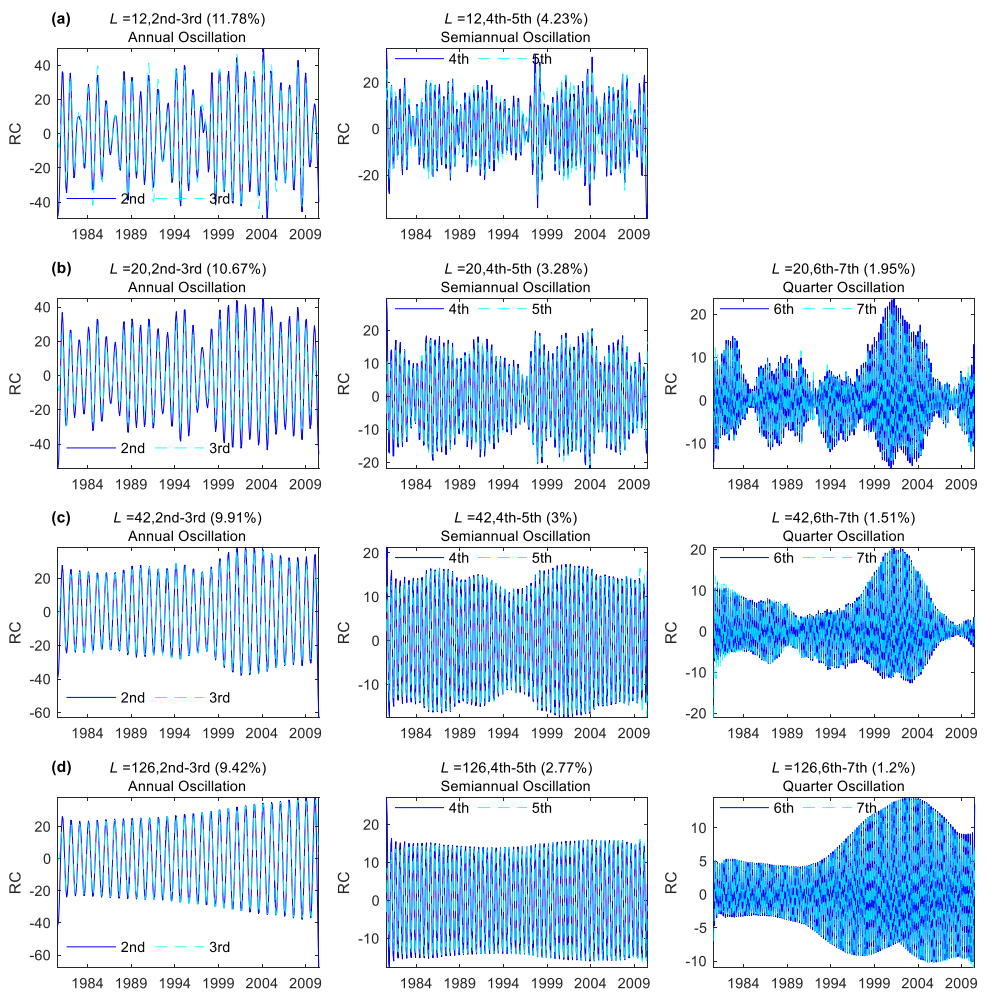

Figure 5. Annual, semiannual and quarter oscillation components are reconstructed from components pairs with $L$ value as $12,20,42$ and 126.
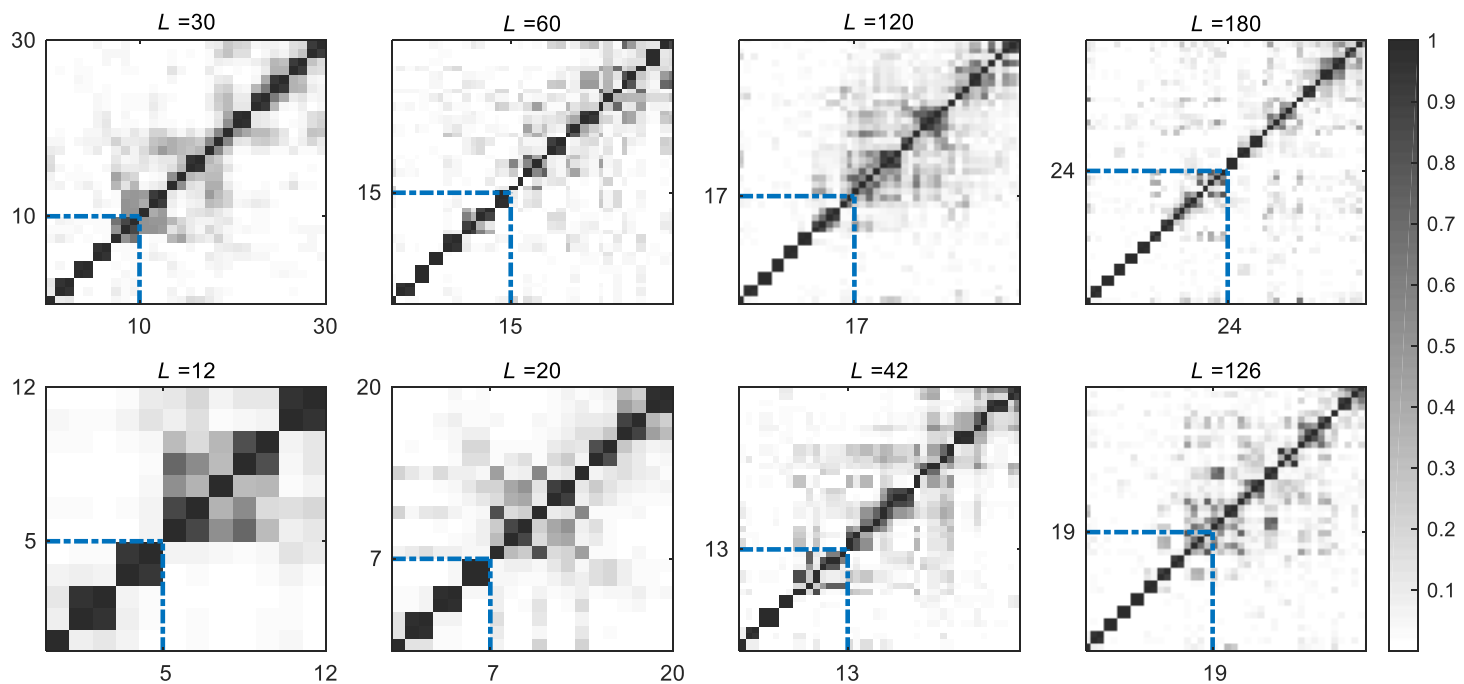

Figure 6. Absolute values of $w$-correlation for reconstructed components (Large values mean high correlation 

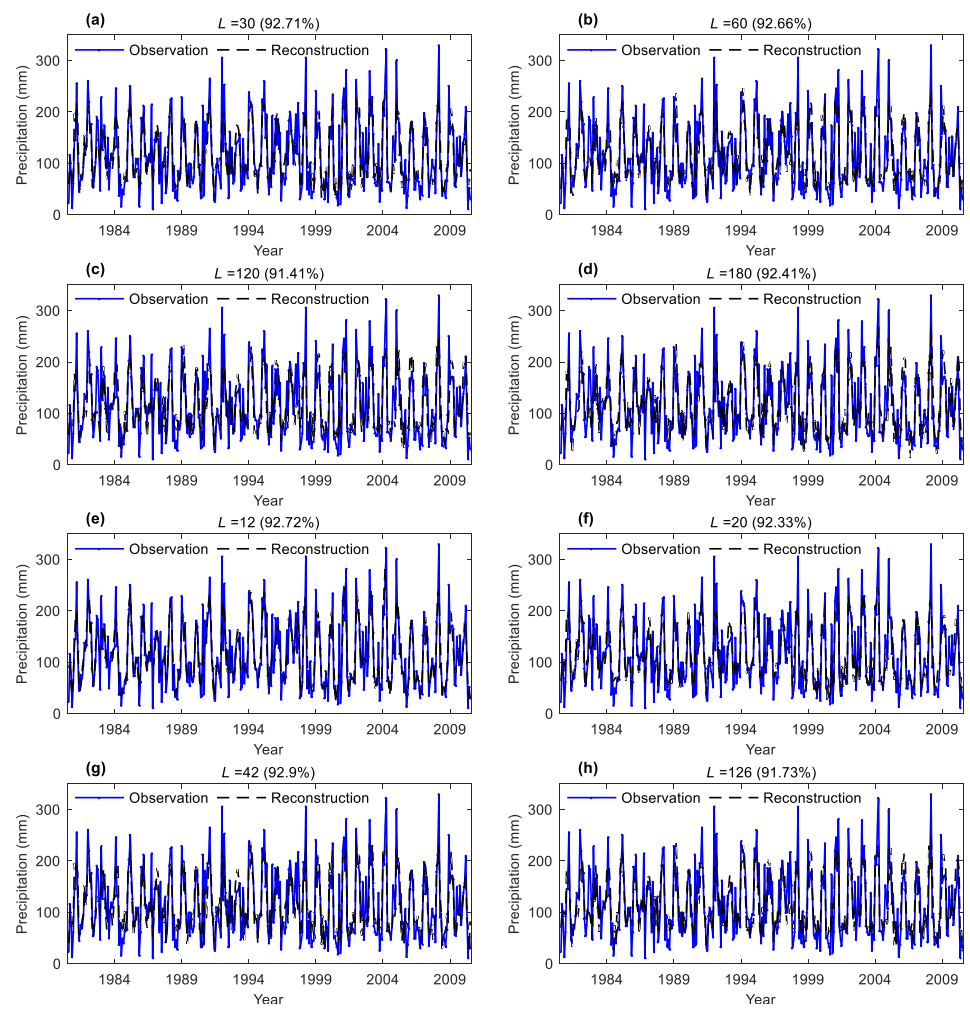

Figure 7. Observation and approximated reconstructions
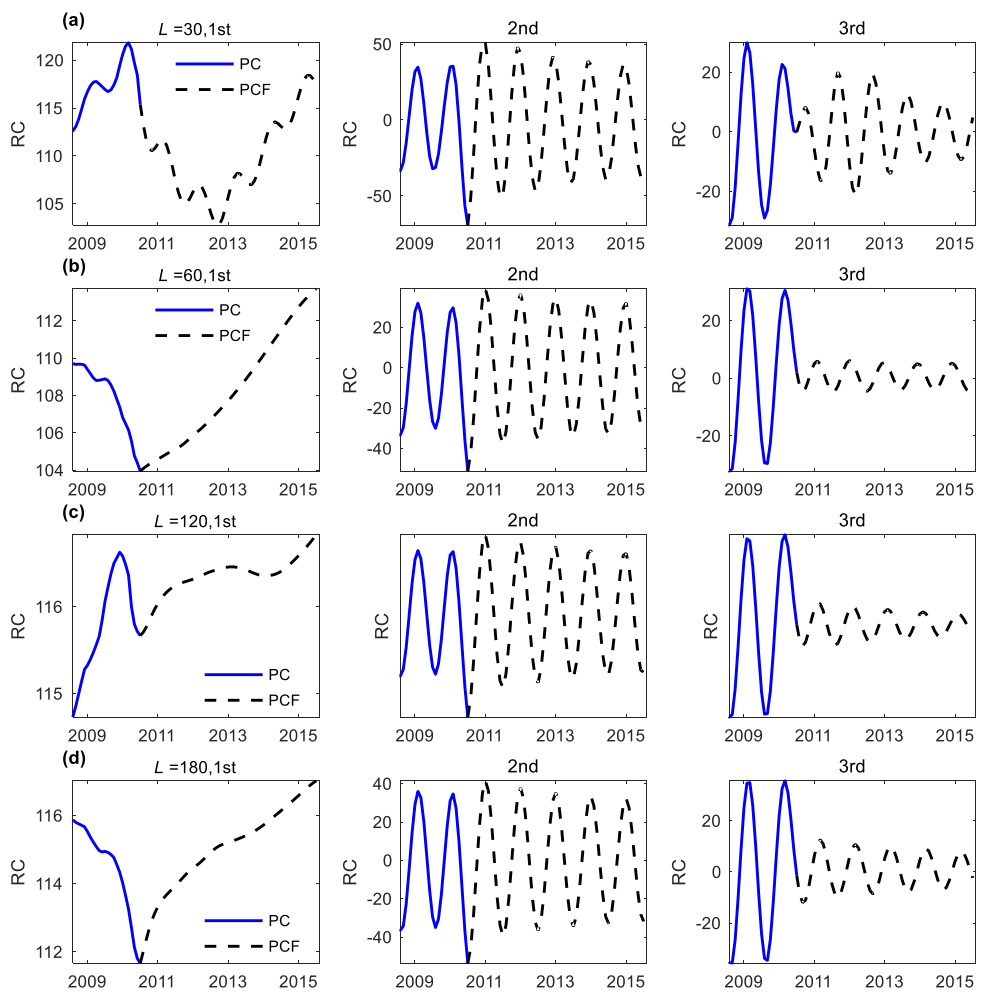

Figure 8. Forecasts of sample leading components time series with L value as 30, 60, 120 and 180

Fig. 7 illustrates the approximated reconstructions of these leading components with different window lengths, as well as the original time series. It can be seen that the reconstruction time series fit the original time series very well except for some peak values. The summary of these reconstructions is shown in Table 1 . On different window length Table 1 shows the leading components numbers, contribution percent of the leading components in all components and the RMSE between original and reconstructions data series. 


\subsection{Forecasting}

Based on the leading component time series, LRF is applied respectively to the time series of those extracted components. Normally, the key parameter $d$ in the Linear Recurrent Formula is decided on experience or repeatedly tried with varying value. After experiment many different sets of value, we took it as same as window length value in this paper. So, the prediction of component is derived through these prepared LRFs, and then these new components are summed up to forecast the precipitation of 5 years (2011-2015). Figs. 8 and 9 demonstrate some sample time series of the leading components with different window lengths. These component series predictions are fairly similar to the variation trend and periodic oscillation of the previous components.
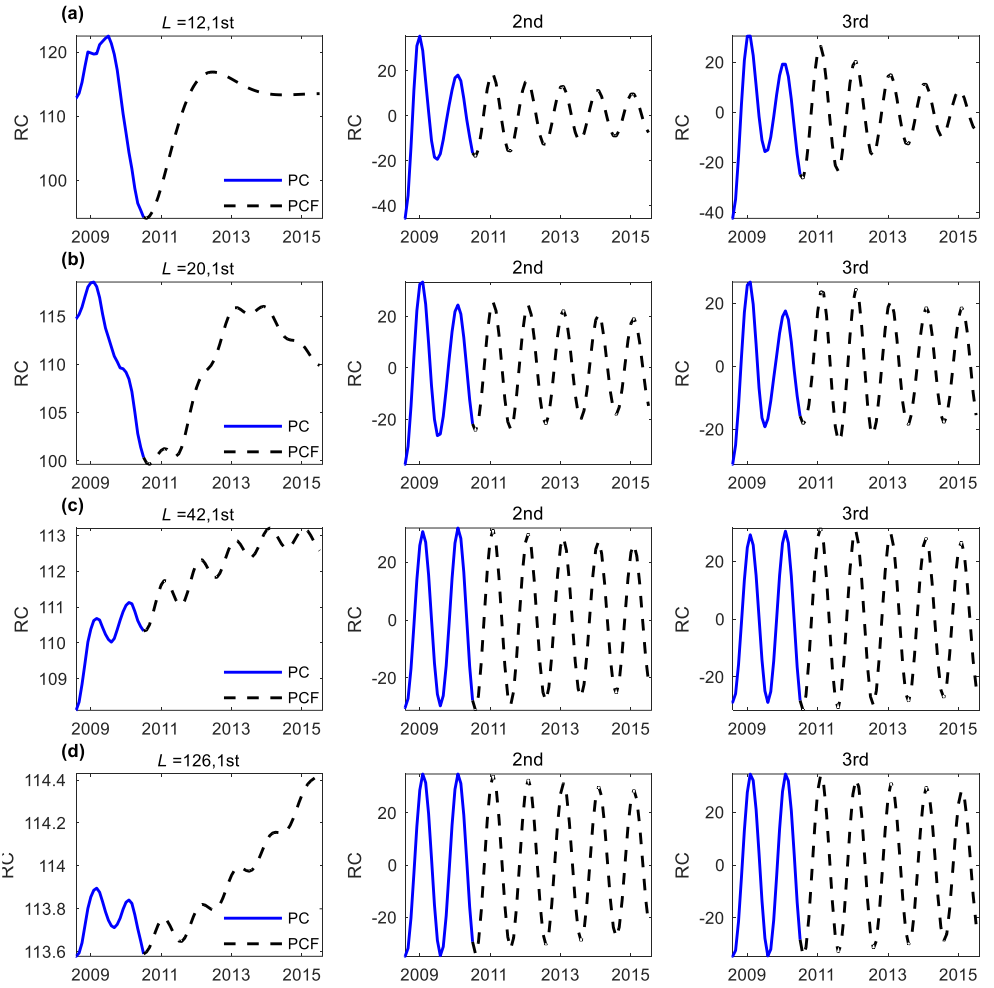

Figure 9. Forecasts of sample leading components time series with $L$ value as $12,20,42$ and 126

According to the prediction of leading components, the precipitation forecasting can be acquired as shown in Fig.

10. Meanwhile, scatter plots of the correlation between forecasts and observation are given in Fig. 11. It is shown that the all regression coefficients are less than 1 , that means the precipitation forecasts are a little underestimate of the observation. Generally the two figures adequately indicate that the forecasts are approximated to the observation, and match well with the variation trends and evolution characteristics of observed precipitation.

With different window lengths, some statistical quantitative verification measures of forecasts are calculated to evaluate their performance according to root mean squared error (RMSE), the coefficient of correlation $(R)$, coefficient of determination $\left(R^{2}\right)$ and mean absolute error (MAE). Table 2 presents the statistical verification contrastive results for these window lengths.

Table 2. Statistical quantitative verification measures of forecasts

\begin{tabular}{c|c|c|c|c}
\hline $\begin{array}{c}\text { Window } \\
\text { Length }\end{array}$ & $\begin{array}{c}\text { RMSE } \\
(\mathrm{mm})\end{array}$ & $\mathrm{R}$ & $\mathrm{R}^{2}$ & $\begin{array}{c}\mathrm{MAE} \\
(\mathrm{mm})\end{array}$ \\
\hline 30 & 41.76 & 0.85 & 0.65 & 30.33 \\
60 & 37.92 & 0.86 & 0.72 & 29.06 \\
120 & 39.53 & 0.84 & 0.69 & 30.53 \\
180 & 41.86 & 0.81 & 0.65 & 32.45 \\
\hline 12 & 53.86 & 0.76 & 0.43 & 38.81 \\
20 & 46.80 & 0.81 & 0.57 & 33.12 \\
42 & 39.02 & 0.85 & 0.71 & 29.58 \\
126 & 38.69 & 0.85 & 0.70 & 29.66 \\
\hline
\end{tabular}

In these standards of verification, it can be found that the forecasts with window length of $L=60$ and $L=42$ both achieve a good result and that their skill measures are 
close. The efficacy of the forecasts with window length $L=60$ is best in all forecasts. These results fully indicate
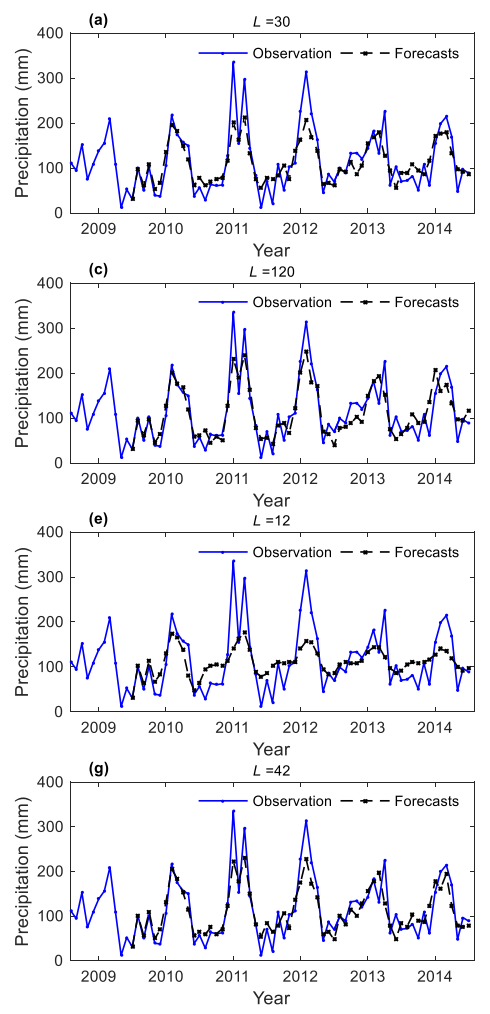

that the SSA-LRFs model has the commendably simulating efficiency on the precipitation forecasting.
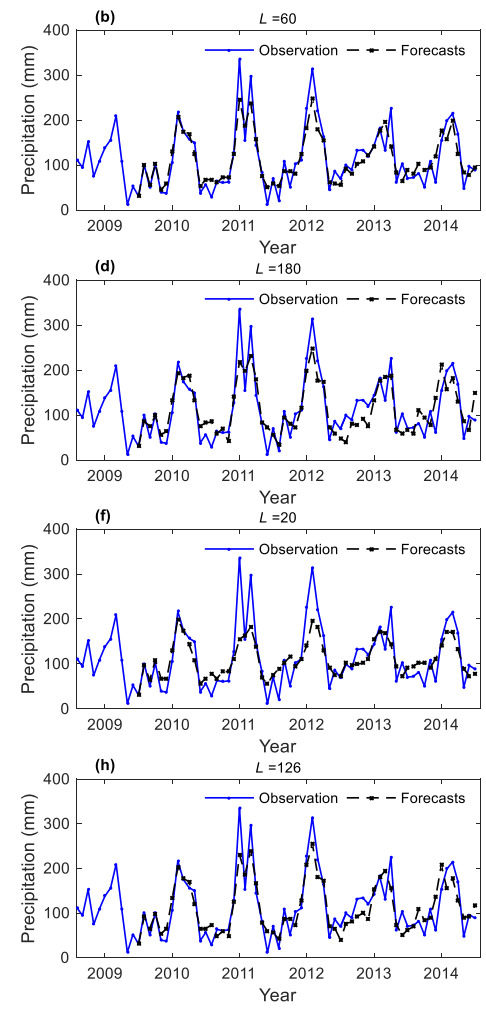

Figure 10. Contrast figures of observation and forecasts
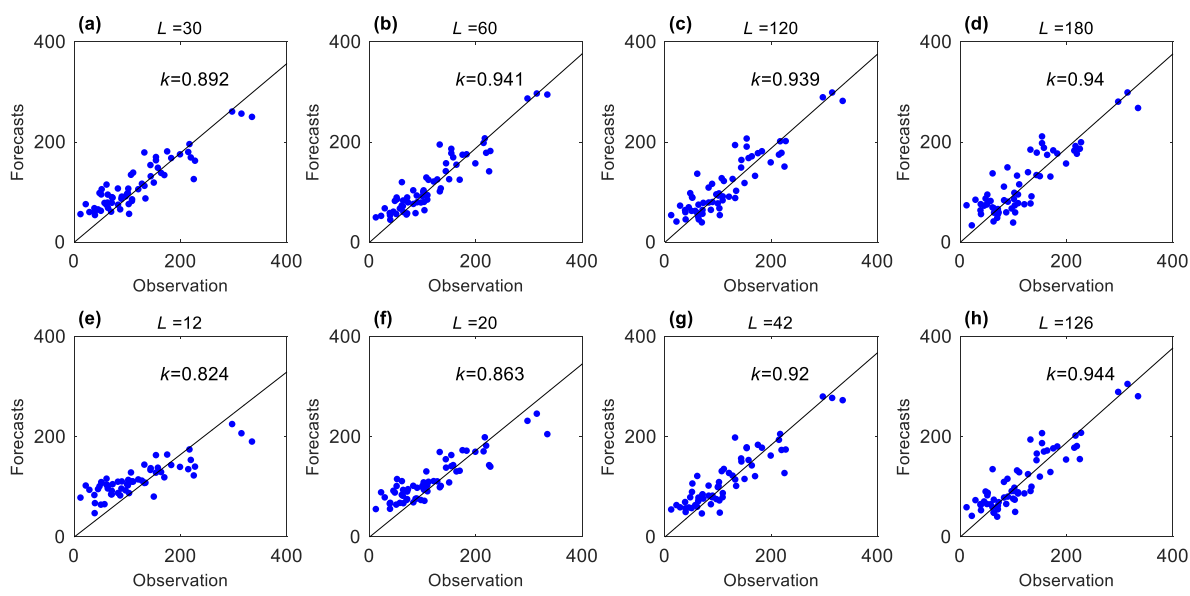

Figure 11. Scatter plots about the correlation of forecasts and observation

\section{Conclusions}

In summary, the variability of precipitation in Florida has been extracted and forecasted successfully using SSA and LRF with different window lengths. Periodogram analysis of precipitation time series shows that some possible inherent periods can be considered as the options of window length. Several different window lengths are selected and the SSA is applied to the tested data. The decomposed components present the characteristics of annual, semiannual and quarterly oscillation. The determined leading reconstruction components are well separated from a block of residual components, and the approximated reconstructions with these leading components fit the original time series very well except for some peak values. Further, LRF is applied to these leading components following the SSA technique with different window lengths, and the comparison of forecasting results with observed precipitation indicates that the forecasts with window length of $L=60$ have the better performance among all. It is indicated that the larger windows lengths $L$ is not always better in SSA application. Our findings successfully confirm that the periodogram analysis can provide a good option for 
window length selection in the application of SSA. It is capable of analyzing and forecasting the seemingly complex time series with potential separable structure, and particularly suitable for forecasting seasonal precipitation variations. As with all analytic methods there are limitations: highly dependent of the periodic signal characteristic and not amenable to the problem requires adaptive algorithms. In the future research, constant effort may be taken to improve the efficiency of model techniques.

\section{References}

Allen M.R. and Smith L.A. (1996), Monte Carlo SSA: Detecting irregular oscillations in the Presence of Colored Noise, Journal of Climate, 9(12), 3373-3404.

Alonso F.J., Castillo J.M.D. and Pintado P. (2005), Application of singular spectrum analysis to the smoothing of raw kinematic signals, Journal of Biomechanics, 38(5), 1085-1092.

Baratta D., Cicioni G., Masulli F. and Studer L. (2003), Application of an ensemble technique based on singular spectrum analysis to daily rainfall forecasting, Neural Networks, 16(3-4), 375-387.

Broomhead D.S., Jones R., King G.P. and Pike E.R. (1987), Singular System Analysis with Application to Dynamica Systems, Chaos Noise \& Fractals, 15-27.

Broomhead D.S. and King G.P. (1986a), Extracting qualitative dynamics from experimental data, Physica $D$ Nonlinear Phenomena, 20(2-3), 217-236.

Broomhead D.S. and King G.P. (1986b), On the qualitative analysis of experimental dynamical systems, Nonlinear Phenomena \& Chaos, 113-144.

Chau K.W. and Wu C.L. (2010), A hybrid model coupled with singular spectrum analysis for daily rainfall prediction, Journal of Hydroinformatics, 12(4), 458-473.

Chen X.Y., Chau K.W. and Busari A.O. (2015), A comparative study of population-based optimization algorithms for downstream river flow forecasting by a hybrid neural network model, Engineering Applications of Artificial Intelligence, 46(PA), 258-268.

Elsner J.B. and Tsonis A.A. (1996), Singular Spectrum Analysis: A New Tool in Time Series Analysis, Springer Berlin, 1283(4), 932-942.

Ghil M. and Vautard R. (1991), Interdecadal oscillations and the warming trend in global temperature time series, Nature, 350(6316), 324-327
Gholami V., Chau K.W., Fadaee F., Torkaman J. and Ghaffari A. (2015), Modeling of groundwater level fluctuations using dendrochronology in alluvial aquifers, Journal of Hydrology, 529, 1060-1069.

Golyandina N. (2010), On the choice of parameters in Singular Spectrum Analysis and related subspace-based methods, Statistics \& Its Interface, 3(3), 259-279.

Golyandina N., Nekrutkin V. and Zhigljavsky A.A. (2001). Analysis of time series structure: SSA and related techniques: CRC press.

Hassani H. (2007), Singular Spectrum Analysis: Methodology and Comparison, Mpra Paper, 5, 239-257.

Hassani H., Mahmoudvand R. and Zokaei M. (2011), Separability and window length in singular spectrum analysis, Comptes Rendus Mathematique, 349(17-18), 987-990.

Hassani H. and Zhigljavsky A. (2009), Singular spectrum analysis: methodology and application to economics data, Journal of Systems Science and Complexity, 22(3), 372-394.

Marques C.A.F., Ferreira J.A., Rocha A., Castanheira J.M., Melo-Gonçalves P., Vaz N. and Dias J.M. (2006), Singular spectrum analysis and forecasting of hydrological time series, Physics \& Chemistry of the Earth Parts $A / B / C, 31(18)$, 1172-1179.

Nourani V., Alami M.T. and Aminfar M.H. (2008), A combined neural-wavelet model for prediction of watershed precipitation, Ligvanchai, Iran, Engineering Applications of Artificial Intelligence, 22(3), 466-472.

Schuster A. (1989), On the investigation of hidden periodicities with application to a supposed 26 day period of meteorological phenomena, Journal of Geophysical Research, 3, 13.

Sun M. and Kim G. (2016), Quantitative Monthly Precipitation Forecasting Using Cyclostationary Empirical Orthogonal Function and Canonical Correlation Analysis, Journal of Hydrologic Engineering, 21(1), 04015045.

Taormina R. and Chau K.W. (2015), Data-driven input variable selection for rainfall-runoff modeling using binary-coded particle swarm optimization and Extreme Learning Machines, Journal of Hydrology, 529, 1617-1632.

Varadi F., Pap J.M., Ulrich R.K., Bertello L. and Henney C.J. (1999), Searching for Signal in Noise by Random-Lag Singular Spectrum Analysis, Astrophysical Journal, 526(2), 1052-1061. Vautard R. and Ghil M. (1989), Singular spectrum analysis in nonlinear dynamics, with applications to paleoclimatic time series, Physica D Nonlinear Phenomena, 35(3), 395-424. 
Vautard R., Yiou P. and Ghil M. (1992), Singular-spectrum analysis: A toolkit for short, noisy chaotic signals, Physica $D$ Nonlinear Phenomena, 58(1-4), 95-126.

Wang W.C., Kwokwing C., Xu D.M. and Chen X.Y. (2015), Improving forecasting accuracy of annual runoff time series using ARIMA based on EEMD decomposition, Water Resources Management, 29(8), 2655-2675.

Wu C.L., Chau K.W. and Li Y.S. (2009), Methods to improve neural network performance in daily flows prediction, Journal of Hydrology, 372(1-4), 80-93.

Yiou P., Baert E. and Loutre M.F. (1996), Spectral analysis of climate data, Surveys in Geophysics, 17(6), 619-663.

Yiou P., Sornette D. and Ghil M. (2000), Data-adaptive wavelets and multi-scale singular-spectrum analysis, Physica $D$ : Nonlinear Phenomena, 142(3-4), 254-290. 